and immune checkpoint inhibitor, but predictive biomarkers are lacking. We performed genomic profiling and characterized specific genes associated with an increased tumor mutational burden (TMB) in Chinese patients with ovarian cancer (OC). OC accounts for high rates of relapse and mortality among people with solid tumors worldwide. As traditional treatments are limited and often intolerant for patients with advanced ovarian cancer, PARP inhibitors and immunotherapy may breakthrough therapies patients immunotherapy could be a better option. In order to explore the potential use of immunotherapy biomarkers, our study aimed to assess gene alteration and its correlations with microsatellite instability (MSI), TMB and tumor neoantigens burden (TNB) in Chinese CLC populations. Methods: A total of 201 female Chinese patients with OC (including primary peritoneal cancer and fallopian tube cancer) were involved from February 2020 to July 2021. All paired specimens were detected by OncoDrug-Seq ${ }^{\mathrm{TM}}$ 603-gene panel assay through next generation sequencing using Illumina NovaSeq 6000. Results: TP53 (62.7\%) was the gene with highest mutation frequency, followed by BRCA1 (18.9\%), KRAS (11.9\%), ARID1A (9.5\%), PIK3CA (9.0\%), NF1 (6.0\%), and BRCA2 (6.0\%). Further analysis showed that TP53 alterations $(84.6 \%, \mathrm{n}=11 / 13)$ were significantly more common in high-grade serous carcinoma than other histological subtypes. Variations detected included fusions $(1.4 \%)$, duplication ( $4.5 \%)$, and deletion ( $4.0 \%)$. Among them the fusions were RET-CCDC6, BRAF-KIAA1549, NRG1-DIP2B, ERBB2MACROD2, especially, the latter 2 fusions occurred simultaneously in one patient. Mutational incidences of significant pathway were also analyzed. Five signal pathways, including JAK-STAT (1.4\%), MAPK (74.6\%), EGFR (28.9\%), WNT (63.7\%), and TGFB $(1.0 \%)$ were related to mutational incidences. Correlation analysis between TMB and mutation status in OC $(\mathrm{n}=110)$ showed that the OC with RAD50 $(n=4)$ or PALB2 $(n=3)$ mutation patterns had higher TMB (mean values 20.3, 25.5, respectively, $\mathrm{p}<0.05$ ), while the MSI status showed no significant difference ( $p>0.05)$. Conclusion: The landscape of mutation patterns and MSI, and TMB among Chinese OC populations in this study will further assist the utilization of these biomarkers to immunotherapy strategies.

Poster (012)

Rare Tumors \& Metastatic Tumors

https://doi.org/10.3802/jgo.2021.32.S1.012

Mucinous ovarian cancer metastasis to cervix: a rare case report

Gatot Purwoto, Raditya Perdhana, Tantri Hellyanti, Trifonia Pingkan Siregar, Siti Rafiqah Fajri
Faculty of Medicine, Indonesia University, Jakarta, Indonesia (raditya.perdhana@gmail.com)

Objective: Ovarian cancer is the second most common gynecological malignancy, but the most lethal. Mucinous ovarian cancer (MOC) is a rare subtype of epithelial ovarian carcinoma. MOC was believed to constitute around $12 \%$ of ovarian malignancies. However, recent estimations show the true incidence to be at around 3\%. it is difficult to diagnose multiple malignancies in genital organ due to almost similar spreading pattern.

Methods: Women, 45 years old with vaginal bleeding and abdominal enlargement. The diagnose of the malignancies was made from Histopathology and advanced imaging. This is a rare case of MOC spreading to cervix has been treat as double primary cancer with radiotherapy and chemotherapy. Results: After completion the therapy (radiotherapy and chemotherapy), patient shows good response without no evidence of disease. the close follow up are need to early detection of the recurrence.

Conclusion: Radiotherapy and Chemotherapy in this rare case of MOC that spread to cervix can treat both for locally advanced cervix metastasis and MOC. A good modality for diagnose is a key to detect a pathologic disease in gynecology malignancies.

Poster (013)

Epithelial Ovarian Cancer including Borderline Tumor https://doi.org/10.3802/jgo.2021.32.S1.013

\section{Predictors of outcome for relapsed epithelial ovarian cancer}

Rudrika Chandra, Neerja Bhatla, Sachin Khurana, MD Ray, Sarita Kumar, Lalit Kumar, Prabhat S. Malik, Sanjay Thulkar All India of Medical Sciences, New Delhi, India (rudrika21@gmail.com)

Objective: To determine the predictors of overall survival (OS) in relapsed epithelial ovarian cancer (EOC).

Methods: Case records of patients with EOC treated between January 2010 and December 2019 were reviewed to identify cases with relapse who received treatment. Primary treatment included upfront cytoreductive surgery followed by adjuvant paclitaxel + carboplatin in 116 (45.7\%) patients and neoadjuvant chemotherapy followed by interval cytoreduction in $138(54.3 \%)$ patients. Following first relapse, 215 (84.6\%) received salvage chemotherapy, 13 (5.1\%) underwent surgery while 27 (10.6\%) patients with biochemical relapse were kept on observation. An intent to treat analysis was done.

Results: Total 254 patients, median age 50 (range $=18-81$ ) years had relapse; median relapses-2 (range=1-9). Median progression 
free survival (PFS1) from diagnosis was 19.3 months (95\% confidence interval $[\mathrm{CI}]=17.9-20.6$ months). Median OS was 50 months ( $95 \% \mathrm{CI}=42.6-57.4$ months). On univariate analysis, PFS1, primary surgery, optimal cytoreduction, complete response to primary therapy, 3 weekly chemotherapy and PFS1 interval were important predictors of OS. On multivariate analysis-response at primary treatment (CR vs. non-CR, hazard ratio $[\mathrm{HR}]=0.47[95 \% \mathrm{CI}=0.29-0.74], \mathrm{p}=0.001)$, type of relapse (clinical vs. biochemical, HR=1.74 [95\% CI=1.19-2.52], $\mathrm{p}=0.004$ ), treatment at relapse (observation vs. chemotherapy, $\mathrm{HR}=1.09[95 \% \mathrm{CI}=0.99-3.6], \mathrm{p}=0.05)$, type of chemotherapy at relapse (paclitaxel + carboplatin vs. non-paclitaxel + carboplatin, $\mathrm{HR}=0.57[95 \% \mathrm{CI}=0.36-0.91], \mathrm{p}=0.018)$ and response to relapse-1 treatment (progressors vs. non progressors, $\mathrm{HR}=2.5$ [95\% CI=1.64-3.8], $\mathrm{p}<0.001)$ were predictors of OS. Conclusion: Response at primary treatment and chemotherapy response at relapse are important predictors of OS.

Poster (014)

Epithelial Ovarian Cancer including Borderline Tumor https://doi.org/10.3802/jgo.2021.32.S1.014

\section{Prognostic value of baseline and 3 months postoperative circulating tumor DNA (ctDNA) in ovarian cancer patients}

\section{Yoo-Na Kim, ${ }^{1}$ Jinho Heo, ${ }^{2}$ Jung-Yun Lee, ${ }^{1, *}$ Seung-Tae Lee, ${ }^{2}$ Saeam Shin, ${ }^{2}$ Sunghoon Kim ${ }^{1}$}

'Department of Obstetrics and Gynecology, Yonsei University College of Medicine, Seoul, Korea (jungyunlee@yuhs.ac)

2Department of Laboratory Medicine, Yonsei University College of Medicine, Seoul, Korea

Objective: Blood sample based circulating tumor DNA (ctDNA) samples are easily obtained and can be used for disease monitoring in ovarian cancer patients undergoing primary surgery.

Methods: Patients undergoing debulking surgery for primary diagnosis or recurrent ovarian cancer were prospectively enrolled since October 2019. Control patients undergoing surgery for benign ovarian mass with CA-125 above 35 were also enrolled. Whole blood samples for cell free DNA analysis were collected immediately before and 3 months after surgery. Custom gene panel covered 9 genes (TP53, BRCA1, BRCA2, ARID1A, KRAS, $M Y C$, and $P I K 3 C A)$. Prepared libraries were sequenced using NextSeq550Dx System (Illumina) and analyzed using the custom analysis pipeline (Dxome). Clinical information was also obtained. Results: A total of 170 patients including 78 patients with epithelial ovarian cancer and 92 control patients with benign or borderline ovarian mass were analyzed. None of the control patients showed detectable ctDNA preoperatively. Analysis of baseline sample showed different ctDNA patterns with respect to histological subtypes. Based on the combination of baseline and 3 months follow up ctDNA, ovarian cancer patients were grouped to non-detected ( $\mathrm{n}=18)$, zero conversion $(\mathrm{n}=41)$, and persistently elevated group $(\mathrm{n}=19)$. Analysis of time to progression with Kaplan-Meier curve showed that the persistently elevated group patients had worse prognosis compared to other groups. Conclusion: ctDNA is a highly specific test for detecting ovarian cancer among patients with suspected ovarian mass on preoperative radiological and biomarker-based evaluation. Despite the variation in mutational landscape among histological subtypes, analysis of baseline and 3 months follow up ctDNA demonstrates a prognostic value.

Poster (015)

Gynecologic Pathology, Genetics and Epidemiology

https://doi.org/10.3802/jgo.2021.32.S1.015

\section{Putative N-glycoprotein markers of ovarian cancer from $\mathrm{N}$-glycoproteomics characterization of the whole cell lysate}

\author{
Ying Zhou, ${ }^{1}$ Zhengjian Feng, ${ }^{2}$ Nengming Lin ${ }^{1, *}$ \\ 'Hangzhou First People's Hospital, Hangzhou, China (Inm03@26.com) \\ ${ }^{2}$ Nanjing Medical University, Nanjing, China
}

Objective: To study the comparative $\mathrm{N}$-glycomics of $\mathrm{N}$-glycans on the cell-surface of ovarian cancer SKOV3 cells vs. the noncancerous ovarian epithelial IOSE80 cells.

Methods: Both SKOV3 and IOSE80 cells were cultured and extracted the proteins. With sequential digestion by trypsin, enriched by Zwitterionic Hydrophilic Interaction chromatography (ZIC-HILIC), the intact $\mathrm{N}$-glycopeptides were then isotopic diethyl labeled and analyzed using C18-RPLC-MS/MS (HCD). Comprehensive structure- and site-specific $\mathrm{N}$-glycoproteomics characterization for both the SKOV3 and IOSE80 cells were searched by GPSeeker database.

Results: Totally 13,822 intact glycopeptides from 2,918 $\mathrm{N}$-glycoproteins with comprehensive structure and site information were identified. A total of 3,733 peptide backbones and 3,754 N-glycosites were confirmed by information of GlcNAccontaining site-determining ions and structure-diagnostic. Seven hundred forty-six differentially expressed intact $\mathrm{N}$-glycopeptides in SKOV3 relative to IOSE80 cells was found, where 421 were upregulated and 325 were downregulated, respectively. Conclusion: Linking observation of differentially expressed $\mathrm{N}$-glycosylation as well as specific N-glycan substructures with epigenetic functions in ovarian cancer might help to develop the novel markers applied in diagnosis and prognosis of ovarian cancer. 